\title{
Astrometry with SIM PlanetQuest
}

\author{
M. Shao \\ Jet Propulsion Laboratory, California Institute of Technology, Pasadena,CA 91007, USA \\ email: Michael.Shao@jpl.nasa.gov
}

\begin{abstract}
SIM PlanetQuest is a very high accuracy space astrometric instrument based on a long baseline stellar interferometer. For global astrometry SIM was designed to be accurate to $\approx 4 \mu a s$ (microarcsec) after a 5 year mission. For narrow angle astrometry $(\approx 1000 \mathrm{~s}$ integration over a $1^{\circ}$ radius field) SIM is designed for $1 \mu$ as precision. The technology program was completed in 2005 and based on laboratory results, the current best estimate of SIM's performance would be $0.6 \mu$ as for narrow angle precision and $2.4 \mu$ as for global accuracy. This paper describes a variety of science programs that the SIM science team have proposed to conduct from a search for one Earth mass planets in the habitable zone of $(\approx 130)$ nearby stars to the study of dark matter in the galactic disk, the galactic halo and the local group.
\end{abstract}

Keywords. techniques: interferometric, astrometry, space vehicles, planetary systems

\section{Introduction}

SIM PlanetQuest is a long baseline optical interferometer designed for ultra-high accuracy astrometry. This paper describes technology status of the SIM project and a few of the science objectives of the Mission. Other papers at this conference will describe several key science projects in more detail (see Beichman 2007, Majewski 2007, Chaboyer 2007, Unwin 2007).

SIM's goal was to be able to do global astrometry with $4 \mu$ as accuracy (5- year mission) and narrow angle astrometry with $1 \mu$ as precision over a $\approx 1^{\circ}$ radius field in $\approx 1000$ second measurement. Because this was such a large increase in accuracy over the Hipparcos mission, NASA established a series of eight technical milestones for the project to accomplish prior to the start of building flight hardware. The next section of this paper is a brief summary of these technology milestones, which were completed in 2005-2006. The completion of milestones brought to light a capability that was not recognized at the start. The last part of this paper provides a snap shot of the variety of science that is planned for the SIM mission.

SIM PlanetQuest, as shown in Figure 1, consists of a science interferometer and two guide interferometers. The guide interferometers provide spacecraft attitude information at the microarcsec level. The science interferometer moves from star to star to measure the relative angles between the stars. The science interferometer and guide interferometers are tied together with a laser interferometer metrology truss, with picometer precision. A long baseline interferometer converts an angle between the baseline vector and the star direction to a length through the classic equation $\mathrm{X}=\mathrm{S}^{*} \mathrm{~B}$, where $\mathrm{X}$ is the delay position measured by the white light fringe, $\mathrm{S}$ is a unit vector to the star and $\mathrm{B}$ is the baseline vector, ${ }^{*}$ is a dot product. Many of the technical challenges come from the need to make these length measurements to very high precision. Two stars $15^{\circ}$ apart would cause the delay $(\mathrm{X})$ to change by $\approx 2.3$ meters. A measurement $2.3 \mathrm{~m}$ length with an error of 43 picometers is equivalent to a 1 microarcsec angular error. 


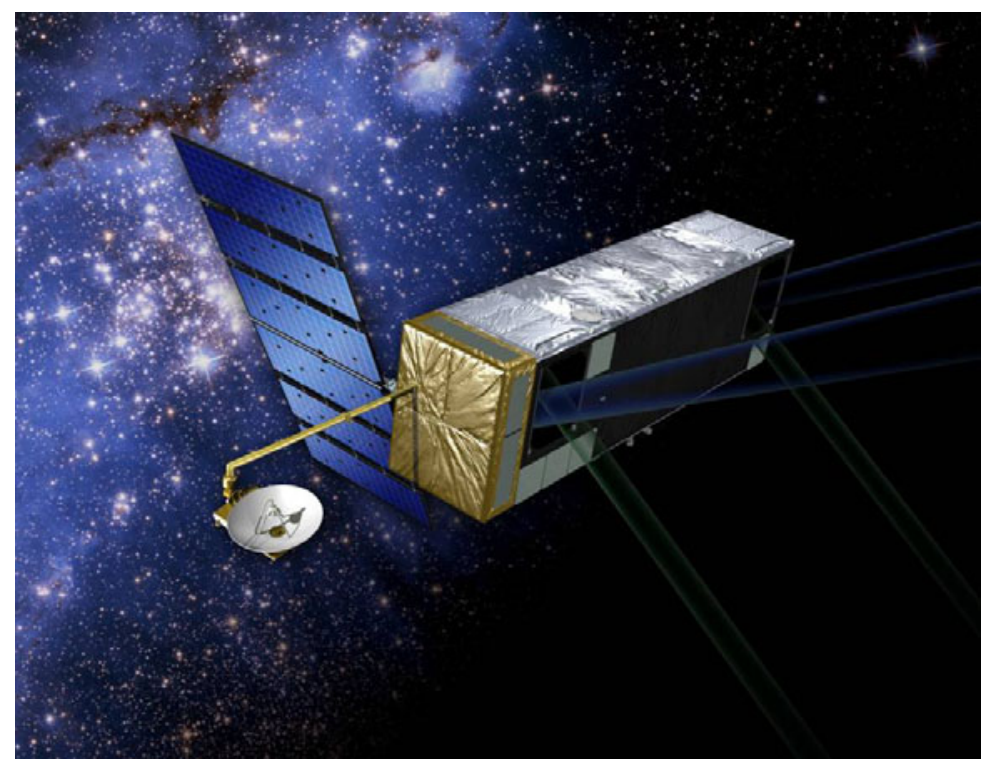

Figure 1. SIM PlanetQuest Spacecraft

\section{Measuring angles to 1 microarcsec}

The eight technology milestones were defined around 2001. The early milestones involved component technologies such as laser metrology gauges that had a precision of a few picometers. Some of the intermediate milestones were system level demonstrations (such as Benchmark No.4 for narrow angle measurements) but at a precision that was 3-5 times worse than the final goal. Four of the milestones represent the critical areas needed for SIM to work in space.

The most difficult tasks concerned the ability to make measurements at the microarcsec level. Since no one has flown a long baseline interferometer in space before, we also have requirements to actively control a large flexible space structure with $\approx 10$ nanometer stability and to point the interferometer (track the white light fringe) with a precision of $\approx 0.1$ milliarcsec. This technology was demonstrated in 2004, with a 3 baseline interferometer and metrology linking the three interferometers. For microarcsec astrometry, the most relevant milestones were the $4 \mu$ as precision for wide angle measurements and the $1 \mu$ as precision for narrow angle measurements.

\section{Global and narrow-angle astrometry}

SIM does global astrometry by measuring the relative position of stars over a $15^{\circ}$ field, which we call a tile, then covering the sky with overlapping tiles. Typically adjacent tiles overlap by 50 percent. In global astrometry the plan is to cover the sky with overlapping tiles $\approx 20$ times over the 5 year mission.

For a technology demonstration, a Micro Arcsecond Metrology testbed(MAM) (see Hines, et al. 2003) was constructed, which includes a test interferometer and a pseudo star, which is an interferometer in reverse. The pseudo star could be moved over $15^{\circ}$ with laser metrology that would measure this motion within $15^{\circ}$. The test setup is shown in Figure 2.

Global astrometry requires measurements over the full $15^{\circ}$ field, while narrow angle measurements were over a $1^{\circ}$ field. The major challenge in global astrometry was to 


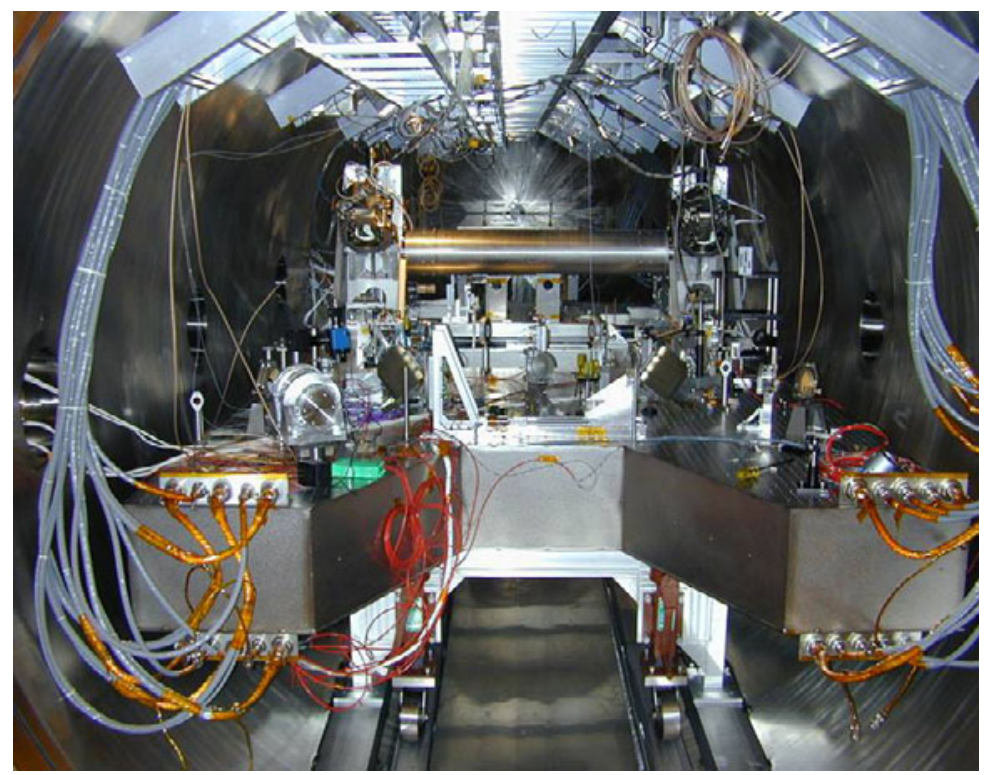

Figure 2. MAM testbed in vacuum chamber

control or model field dependent biases to $4 \mu$ as. Concurrent with the laboratory effort was a numerical simulation activity that looked at taking many single tile measurements over the whole sky over 5 years and performing a large least-squares fit of the global parameters in order to derive the position, proper motion and parallax of all objects. In doing the global astrometry simulations several properties were discovered. As suspected a linear scale error in the single tile measurements was not important, as long as they were relatively small. A number of higher order field dependent biases, could be "solved" for in the grid solution if these errors were static over a period of several days. The wide angle astrometry milestone was met in 2005 .

The narrow angle milestone was in some way more difficult because the goal was $1 \mu$ as in $\approx 20$ minutes instead of $4 \mu$ as after 5 years. But the small field $\left(1^{\circ}\right)$ means that the major concern in global astrometry, i.e. field dependent biases, was no longer a concern. The major error for narrow angle astrometry turned out to be thermal drift. The laser metrology beam is placed in the center of the white light beam so to first order any motion of optical elements would be monitored. However there were numerous second order effects.

Figure 3 shows the results of the narrow angle tests. For the SIM instrument with $9 \mathrm{~m}$ baseline $1 \mu$ as is roughly $50 \mathrm{pm}$ of optical path. But the allocation to the science interferometer was only $28 \mathrm{pm}$. One $\mu$ as (1 sigma) precision means that $>68 \%$ of the measurements must have an error within $\pm 28 \mathrm{pm}$.

Our original plan for exoplanet detection was to visit a target star 25-50 times over 5 years and survey a large number of stars. If we assumed that errors at different epochs were random that was the end of the story. Ideally we should demonstrate in the lab that the systematic error floor was $<1 / \sqrt{25}$. Key to narrow angle measurements is chopping. SIM will move between target and reference star with a $\approx 90 \mathrm{sec}$ period. To demonstrate that the systematic error floor was near $0.2 \mu \mathrm{as}$, we conducted a longer test. Figure 4 is a plot of the Allan variance. For integration times of $\approx 1$ day we had not reached the systematic error floor. The Allan variance had reached 1 picometer in optical path, which 


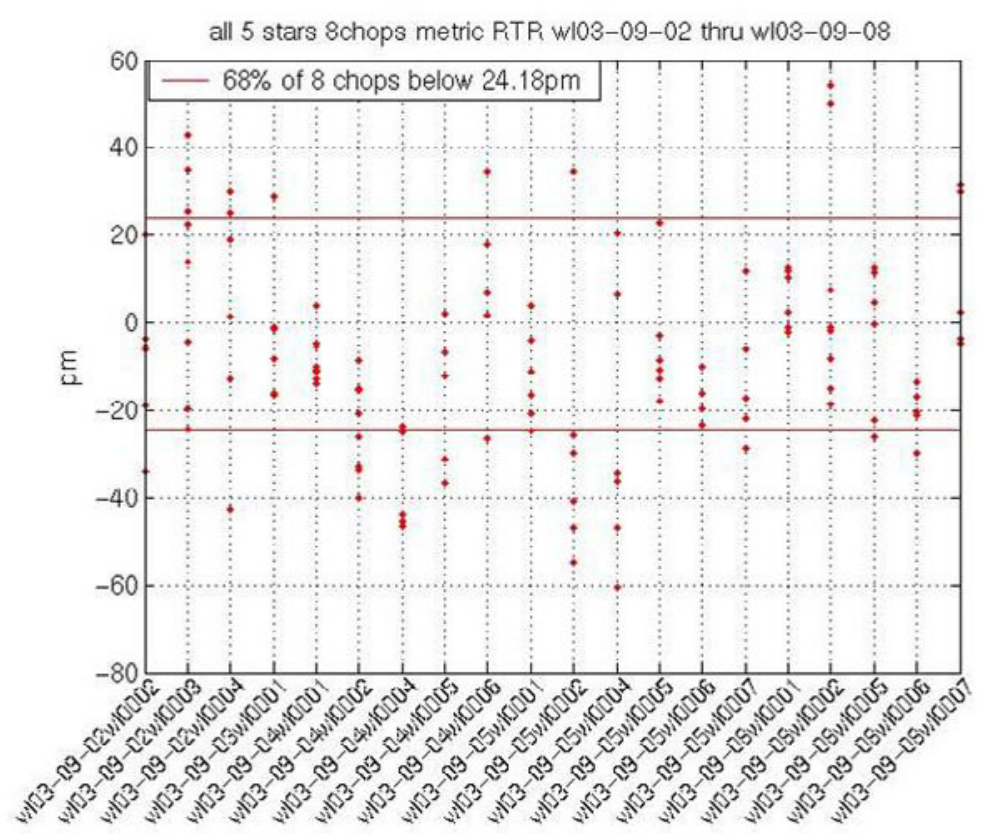

Figure 3. MAM results of 20 narrow angle tests

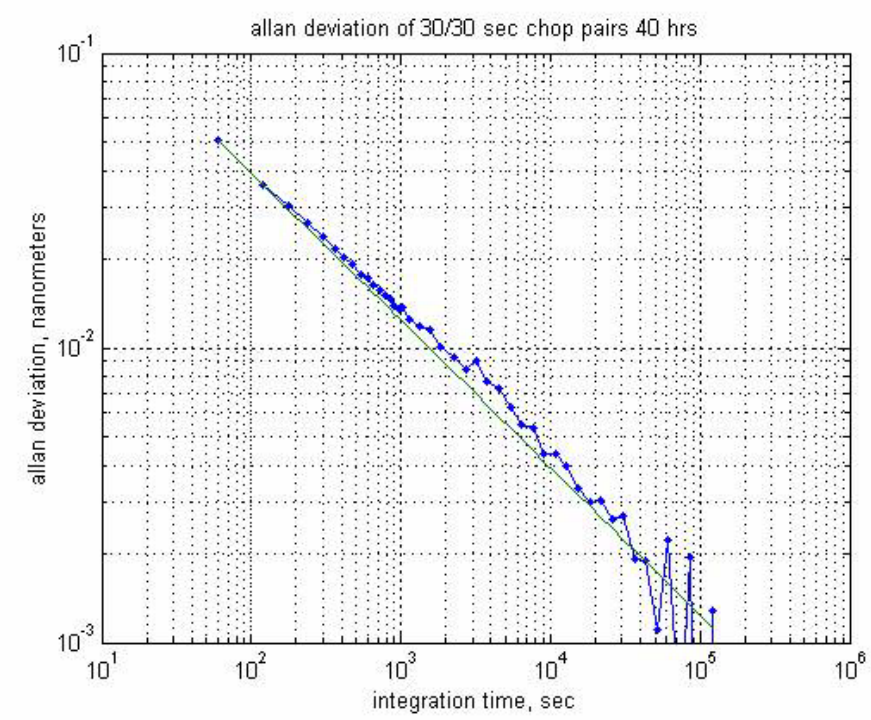

Figure 4. Allan variance of narrow angle measurements

divided by the $9 \mathrm{~m}$ baseline is $0.023 \mu$ as. The very low systematic error floor enables a different type of exoplanet search. 
Table 1. Number of detectable Earth-like planets versus the mission systematic noise floor

\begin{tabular}{cc}
\hline Noise floor & No. of targets \\
\hline $0.2 \mu \mathrm{as}$ & 2 \\
$0.1 \mu \mathrm{as}$ & 4 \\
$0.075 \mu \mathrm{as}$ & 14 \\
$0.05 \mu \mathrm{as}$ & 43 \\
$0.04 \mu \mathrm{as}$ & 80 \\
$0.03 \mu \mathrm{as}$ & 198 \\
\hline
\end{tabular}

\section{Science for searching exoplanets}

Over 250 exoplanets have been discovered. The SIM tier-1 exoplanet project is to look for terrestrial planets in the habitable zone. If we are limited to looking for astrometric signatures $>1.0 \mu \mathrm{as}$, there are only two stars around which an Earth clone $\left(1 M_{E a r t h}, 1\right.$ $A U * \sqrt{L}$, where $\mathrm{L}$ is luminosity) can be found, i.e. two stars of the wide binary $\alpha$ Cen. But if instead of just $\approx 50$ visits per star and a total of 14 hours of observing time in 5 years, we increase the total time to 200-250 hours, the minimum detectable astrometric signature would drop by a factor of $5-6$, and the number of stars around which an Earth clone could be detected would grow substantially. The Table 1 lists the number stars around which an Earth clone can be found versus the mission systematic noise floor.

SIM PlanetQuest with its very low systematic noise floor is limited by the total observing time. The current best estimate (with no margin) is that a target star of 7 mag with 9.6 mag reference stars could be measured with $0.7 \mu$ as in a $1000 \mathrm{~s}$ measurement. Getting to a mission accuracy of $0.03 \mu$ as means over 150 hours spent per target. One hundred such targets would occupy two full years of integration. If we were to conduct a search down to $1 M_{\text {Earth }}, 1 \mathrm{AU}$, the nearest stars would not need 150 hours each. But the majority of the stars would. Going through the list of nearest stars, SIM PlanetQuest can conduct a search of $\approx 130$ stars for an Earth clone, using $40 \%$ of its 5 year mission.

While we have demonstrated that instrumental errors can be controlled at levels of well below $1 \mu$ as, we need to take a careful look at astrophysical noise or error sources. Two major astrophysical errors are: 1) star spots or more generally, stellar variability that results in a displacement of the center of light with respect to the center of mass, and 2) the presence of planets around the reference stars used for narrow angle astrometry. These topics will be addressed in much more details in a separate paper underway. A typical star spot on the Sun at 10 pc that causes a $5 * 10^{-4}$ photometric variation which produces a maximum astrometric shift of $0.12 \mu$ as. Just as important, at periods longer than the 30 day rotation period of the Sun the astrometric noise from star spots averages out (spot on the left drifts to the right side of the star) as $\sqrt{N}$, where $\mathrm{N}$ is the number of epochs where the separation between epochs of observation is greater than the rotation period of the star. In our simulations, putting in an 11 year sunspot cycle shows no astrometric noise with an 11 year period.

The second astrophysical concern is planets around the reference stars. The reference stars are typically 9-10 mag $\mathrm{K}$ giants at a distance of 700-1000 pc. A Jupiter in a $1 \mathrm{AU}$ orbit around a one solar mass $\mathrm{K}$ giant would have an astrometric wobble of 1 $\mu$ as. If the instrumental noise floor was $0.03 \mu$ as, these planets with a $1 \mu$ as signature are easily detected and their effect can be removed from the "local" reference frame. Much smaller planets $(<0.05 \mu \mathrm{as})$ would be simply absorbed as noise. Intermediate sized planet (signature between $0.3 \mu$ as and $0.05 \mu$ as) could not be reliably detected but would produce wobbles that are not ignorable. On average only $\approx 5 \%$ of stars would have such 


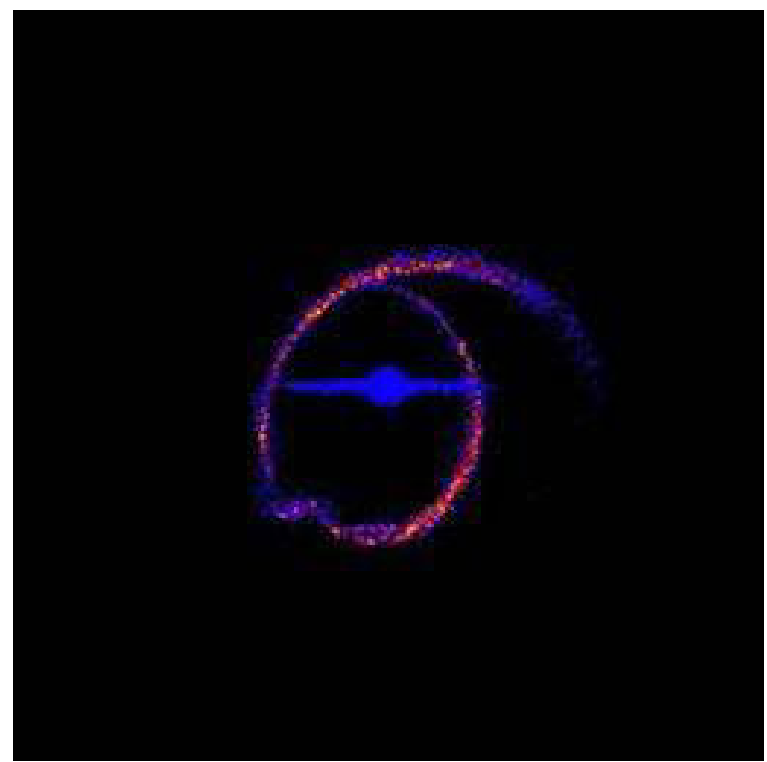

Figure 5. A demonstration of the sensitivity of SIM to the Galactic potential using stellar steams

planets. And if only 1 of 4 reference stars are like this, the end result is only a minor adjustment in the mission noise floor.

\section{Astrophysics with SIM}

When the SIM science program was selected in 2001-2002 through a call for proposals, Gaia was already a well established mission concept under study at ESA. Consequently, most of the SIM astrophysics is in areas outside of the main Gaia science program. The principle area for SIM astrophysics (see Unwin, S. et al. 2007) is moderately high accuracy $(\approx 10 \mu \mathrm{as})$ astrometry of faint stars $(17-20 \mathrm{mag})$.

The major themes for SIM astrophysics are dark matter, Hubble constant, and the masses of post main sequence objects like neutron stars and stellar mass black holes in binaries. Dark matter will be studied by several key projects. One key project with Andrew Gould as the PI would followup microlensing events both astrometrically and photometrically with SIM $(\approx 0.5 \mathrm{AU}$ from the Earth) to tightly constrain the mass of the lensing object. Another with Steve Majewski would measure the parallax and proper motion of faints stars in tidal tails of dwarf galaxies ripped apart by the gravity gradient of the Milky Way. The motion of the stars in the tidal tales will let us map the dark matter distribution in the halo of our galaxy as shown in Figure 5. The third project in this area is the measurement of the proper motion of galaxies in the local group, to get a handle on the dark matter in the local group shown in Figure 6.

Proper motion of stars in a few nearby spiral galaxies will enable us to measure the distances to these galaxies via a dynamical parallax technique. This distance could be used to calibrate the zero point of the Cepheid period-luminosity-metalicity relation.

Last of all SIM will be able to measure the orbits and hence masses of neutron star and black hole binaries throughout the galaxy. Many of these object are both faint and have short periods which require a short but concerted observing campaign. 


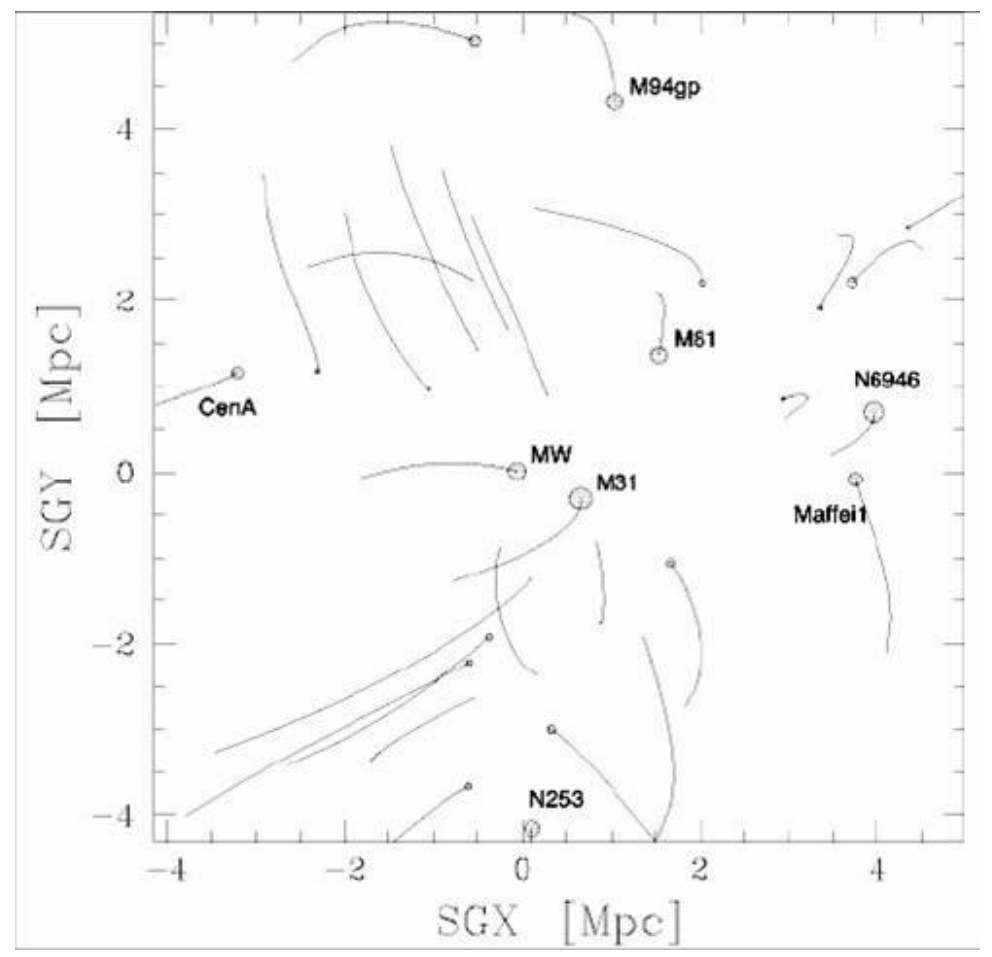

Figure 6. The Trajectories of nearby galaxies and groups going out to the distance of the Virgo Cluster from a NAM calculation

\section{Summary}

SIM Planet Quest is a mission whose very ambitious technology has been completed after a decade of effort. This technology will enable a wide range of science from the detection of Earths around nearby stars to the study of dark matter in the halo of our galaxy.

The research described in this paper was carried out at the Jet Propulsion Laboratory, California Institute of Technology, under a contract with the National Aeronautics and Space Administration.

\section{References}

Beichman, C. 2008, Proceedings of IAU Symposium 248, in this volume p.238 Chaboyer, B. 2008, Proceedings of IAU Symposium 248, in this volume 1.440 Hines, B., et al. 2003, SPIE, vol. 4852, p45

Majewski, S. 2008, Proceedings of IAU Symposium 248, in this volume $p .450$

Unwin, S. 2008, Proceedings of IAU Symposium 248, in this volume p.288

Unwin, S. et al. 2007, PASP, accepted 